EOMmunutriln? Communication et organisation

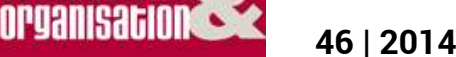

Design \& projet

\title{
Data design-moi un mouton
}

De la data visualisation au data storytelling chez Michael Paukner

\section{Vivien Lloveria}

\section{(2) OpenEdition}

\section{Journals}

Édition électronique

URL : http://journals.openedition.org/communicationorganisation/4747

DOI : 10.4000/communicationorganisation. 4747

ISBN : 979-10-300-0155-6

ISSN : 1775-3546

\section{Éditeur}

Presses universitaires de Bordeaux

\section{Édition imprimée}

Date de publication : 1 décembre 2014

Pagination : $99-112$

ISBN : 978-2-86781-905-6

ISSN : 1168-5549

Référence électronique

Vivien Lloveria, «Data design-moi un mouton », Communication et organisation [En ligne], 46 | 2014, mis en ligne le 01 décembre 2017, consulté le 26 avril 2019. URL : http://journals.openedition.org/ communicationorganisation/4747 ; DOI : 10.4000/communicationorganisation.4747 


\title{
Data design-moi un mouton De la data visualisation au data storytelling chez Michael Paukner
}

\author{
Vivien Lloverio ${ }^{1}$
}

\section{Introduction}

En préambule de l'article de Bertin (1981) Communication et Langages s'interrogeait sur l'état de connaissance de ses lecteurs : "Mais connaissent-ils la graphique et font-ils la différence entre la graphique et le graphisme ? ». La réponse nous suggère un parcours, prenant pour origine la visualisation de données (data-visualization) et comme destination le design (data-design) et le récit des données (data-storytelling). Ce cheminement, nous le ferons au travers d'une œuvre de Michael Paukner, intitulée Dominated by sheep $p^{2}$, présentée sur son site internet et reprise en version française dans Courrier International (Paukner, 2013 : 39).

\section{Questionner le genre}

Le but initial de la graphique de Bertin fut conforme à celui des statisticiens de la fin du XVIII e siècle, à savoir de représenter visuellement des données. Par ce constat historique nous souhaitons déconstruire l'idée reçue qu'elle serait une invention récente, née de l'apparition de l'informatique. Le projet Milestones (Friendly et Denis, 2014) retrace cette épopée dont les premières graines furent semées avant l'ère chrétienne et se seraient développées pendant plusieurs millénaires. Forte de cette longue maturation, la graphique de Bertin se présente comme l'héritière d'un genre, celui de la représentation statistique et cartographique du monde. Dans la tradition greimassienne, le genre est de nature sociolectale (Greimas et Courtès, 1993 : 164), lié au collectif, à une institution qui standardise la signification. Selon Hjelmslev (1971 : 81), le

1 Vivien Lloveria est docteur en Sciences du langage. Il enseigne au département Sciences du Langage de l'Information et de la Communication à l'Université de Limoges. Il mène ses recherches au Centre de Recherches Sémiotiques (CeReS) ; vivien.lloveria@unilim.fr

2 Michael Paukner, Dominated by sheep, http://www.substudio.com/dominated-by-sheep, consulté le 25 avril 2014. 
genre dérive d'un dialogue entre la norme abstraite et ses usages manifestés, ce qui la dynamise et lui procure une relative instabilité. Les habitudes méthodologiques consistent à distinguer un genre d'un autre qui, juxtaposés indéfiniment, forment des classes typologiques dont la profusion se révèle vite problématique. Pour les dépasser, nous adopterons une démarche inspirée par Fontanille, pour qui les genres « établissent un certain rapport entre deux plans d'immanence : d'un côté le texte, de l'autre côté la pratique » (Fontanille, 2008 : 67). Pour résumer sa pensée, les types pratiques se retrouvent incarnés sur la surface textuelle sous la forme d'empreintes qui seront à leur tour reconnues, comme genre, et interprétées comme des instructions pratiques. De cette manière, étudier le genre visualisation de données, consistera à saisir les pratiques collectives fossilisées dans de grands ensembles textuels, dont la diffusion et la circulation ont permis l'émergence. Nous tenterons d'y préciser la nature des instructions de production, d'interprétation mais aussi de contrôle, communiquées par le genre. De cette façon, nous espérons découvrir ce qui guide les démarches d'anticipation, les cadres de régulation inhérents à toute démarche de projet de visualisation, de design et de récit de données. Notre approche sera celle de la sémiotique, privilégiant un sens linguistique (Rastier, 2001 : 106) aux sens « extralinguistiques $»^{3}$, pour mettre au jour la variété de ses conditions d'actualisation. D'un point de vue méthodologique, nous mobiliserons la perspective récente d'une sémiotique des empreintes pour laquelle "l'interprétation est une expérience qui consiste à retrouver les formes d'une autre expérience, dont il ne reste que l'empreinte » (Fontanille, $2011: 180)$.

\section{Visualise-moi une donnée (data-visualization)}

Bertin expose dès le début de son article un certain système de valeur (une axiologie) car bien que la sémiologie graphique existe, il constate que « cependant les cartes et les diagrammes inutiles sont encore très nombreux. Il fallait trouver quelque chose de plus simple pour éviter ces erreurs » (Bertin, 1981). Nous repérons alors un double attachement : celui concernant l'utilité et celui visant la simplicité de la représentation. Pour être utiles, les visualisations doivent autoriser les regroupements visuels et les ordonnancements de manière à conférer à la multiplicité des informations une accessibilité et une lisibilité quasi-immédiate. Pour Bertin, " on ne lit pas un graphique, on lui pose des questions " (Bertin, 1981). Si sa fonction principale est bien de « répondre à des questions » posées par le détenteur des données, elle devient spécifique dès lors qu'elle se présente comme une solution iconique (Fontanille et Dondero, 2012), transformant en informations intelligibles un ensemble de données que le système humain ne peut embrasser. Cette spécificité en fait un informateur diagrammatique destiné à transmettre une information spécialisée

3 Telles que les représentations psychiques (conceptuelles) des approches cognitives, ou les déterminants sociaux des approches sociologiques. 
sur les relations entre données. Son interprétation se particularise aussi puisque « la perception consiste à définir les relations qui s'établissent dans l'image, ou entre images, ou entre images et nature. Le travail de lecture se situe entre les significations »(Bertin, 2013). Une visualisation que l'on questionne, modifie sensiblement le rapport entre observateur et informateur, et la graphique de Bertin s'efforce de transformer le spectateur devant le visuel en un acteur dans le visuel (Bertin, 2013). Si la représentation doit être globalement lisible, elle doit également permettre d'identifier et de distinguer le qualitatif (différences et ressemblances), le quantitatif et l'ordonné. Ces possibilités interrogent à la fois les compétences de l'informateur qui doit se rendre visible, lisible, analysable et mémorisable, mais aussi celle de l'observateur nécessairement doté de compétences de lecture, d'interprétation et de mémorisation liées au genre (ce que nous associons à la littératie). Bertin associe fortement le questionnement sur l'image avec le niveau de compétence de l'observateur (Bertin, 2013 : 39). Ce dernier peut ainsi se demander « quel rapport $y$-a-t-il entre deux longueurs ou deux surfaces ? " (attitude perceptive quantitative), "dans quel ordre ces longueurs sont-elles classées? " (attitude perceptive ordonnée), « où sont les villes de plus de 15000 habitants? " (attitude perceptive sélective) et enfin quelle est la répartition des villes entre 5000 et 15000 habitants (attitude perceptive associative). La visualisation doit refléter le questionnement, en organisant les distinctions, en homogénéisant pour suggérer les associations, en engendrant un ordre et manifestant des quantités. Cette maîtrise du visuel, par des règles partagées constitutives du genre, fut justement l'ambition de Bertin. Dans son univers, " tous les participants s'accordent sur certaines significations, exprimées par certains signes, et conviennent de ne plus en discuter "(Bertin, 2013 : 7). La graphique vise une monosémie visuelle, au moyen d'un système clos et fortement codé, dont l'objet est de détourner des éléments visuels afin de se focaliser sur les relations qu'ils exemplifient.

Bertin insiste alors sur deux questions qui n'ont pas lieu d'être dans la graphique : «Que voyez-vous ? Que préférez-vous?»(Bertin, 1981). Ces deux questions apparemment anodines s'avèrent représenter de précieux leviers pour caractériser les évolutions les plus récentes du genre, à savoir le design et le narratif. Le « que voyez-vous ? " ne concerne pas vraiment l'acuité perceptive, mais renvoie plutôt à un implicite déterminant pour notre démonstration : celui de la capacité à engendrer la reconnaissance. Pour Bertin, la graphique n'est pas là pour représenter des entités du monde naturel, trahissant de la sorte un certain rejet de l'iconisme ou du figuratif. Ce rejet, Bertin le justifie au nom de la monosémie : « la graphique est un système monosémique alors que l'image figurative est polysémique » (Bertin, 2013 : 7). Cette polysémie devient alors menaçante car selon lui, « la signification est personnalisée et discutable» (Bertin, $2013: 7)$. Nous en conclurons qu'un projet conforme à la graphique doit prioritairement anticiper l'ensemble de son lexique visuel, 
de manière à strictement définir une signification indiscutable, pour pouvoir discuter les relations entre unités monosémiques.

\section{Visualise-moi les données sur les moutons...}

Il s'agit maintenant de comprendre comment l'œuvre de Michael Paukner exemplifie, du moins en partie, le genre visualisation de données. Pour commencer, la visualisation est d'emblée assimilable à une catégorie de diagramme dans la graphique de Bertin : la chronique. Cette dernière organise deux séries de données en respectant un certain standard. La première série, celle des données temporelles, reprend l'horizontalité stéréotypique de la plupart des représentations chronologiques, en déroulant une ligne temporelle respectant le sens de lecture gauche/droite institué par les sociétés occidentales. Nous retrouvons les données quantitatives en ordonnée, respectant alors une organisation typique de la verticalité qui impose un sens bas/haut pour les valeurs positives. Enfin, des données qualitatives sont intégrées aux courbes par une distinction chromatique qui, au plan du contenu, différencie les moutons (blanc) des humains (orange). Cependant, toutes ces précautions pour satisfaire le standard de la graphique, sont-elles le fruit de décisions personnelles de la part du designer? Le doute s'installe lorsqu'on examine d'un peu plus près. En effet, l'œuvre signale un lien internet vers une version originale de la chronique, celle précédemment réalisée par la société DataMarket qui " aide les professionnels à trouver et comprendre les données " ${ }^{4}$. Opérant la comparaison, une chose saute aux yeux dans la version de Paukner : elle paraît moins précise que sa version d'origine. En effet, l'abscisse millimétrée de la version DataMarket, laisse place à une version plus grossière n'affichant plus que les quinquennats. Cet effacement des données intermédiaires révèle une volonté d'orienter l'observateur sur une lecture d'ensemble de la courbe en négligeant les autres parcours. Or pour l'inventeur de la graphique, la qualité de la représentation dépend justement de ses possibilités d'exploration. Elle doit en outre permettre de mener une lecture élémentaire destinée à extraire une valeur de la courbe, une lecture moyenne pour découvrir des regroupements de valeurs plus ou moins homogènes et une lecture d'ensemble pour réduire la composante à une unique relation (la tendance générale). Chez Paukner, les valeurs ne sont pas lisibles élément par élément (i.e. nous ne pouvons dire combien il y a de moutons en 1990), nous pouvons à la rigueur repérer des motifs accidentels (i.e. retour cyclique, pic). Cette imposition de la lecture d'ensemble semble être corroborée par les huit petites chroniques placées sous la chronique principale, dont la légende justifie la présence : "Si ce ratio est plus élevé en Nouvelle-Zélande que n'importe où dans le monde, les moutons sont toutefois en surnombre dans plusieurs autres pays ». En conséquence, la fonction des petites chroniques n'est pas tant de nous fournir des détails,

4 Notre traduction d'About DataMarket, http://datamarket.com/p/about/, consulté le 11 avril 2014. 
que d'exposer la répétition d'un même phénomène, à savoir la supériorité numérique des moutons sur la population humaine. Cette intention, dévoilée par le dispositif visuel, confirme notre hypothèse d'un fenêtrage volontaire des données sur la seule relation de domination entre les deux courbes. Qu'importe si les courbes apparaissent éloignées comme en Australie, ou entrelacées comme au Soudan, l'information première à communiquer demeure la position de domination d'une population vis-à-vis d'une autre. Enfin, nous remarquerons comment deux valeurs sont surexposées par le recours à des ronds placés sur la ligne et la communication de leurs valeurs numériques au moyen d'indications textuelles. Pour ces raisons, la durée des années écoulées (en abscisse) et la quantité de la population (en ordonnée) sont reléguées au rang de données secondaires constituant ainsi l'arrière-plan de la démonstration-ostentation anticipée par le designer.

Nous identifions un autre indice de manipulation dans la différence notable, entre la forme d'ensemble de la chronique élaborée par DataMarket et celle de Paukner. Dans la version de DataMarket, la courbe ne présente qu'une tendance décadente depuis son pic de 1982, soulignant une chute progressive et régulière des moutons. Bien différemment, une telle évidence iconique de la chute de la population ovine demeure absente de la courbe de Paukner. Son œuvre semble atténuer cette décadence, voire tenterait même de nous faire croire au maintien des effectifs par l'insistance de l'horizontalité. Aussi, prenant à contre-pied l'injonction de vérité de la graphique, le travail de Paukner serait à considérer, au mieux, comme une maladresse, au pire, comme un dévoiement volontaire des données. Manifestement, Paukner fragilise l'idéal de vérité et de simplicité de la graphique pour servir d'autres intentions que celles qui contraignent le genre. D'une manière quelque peu triviale, nous constatons que cette version est simplement plus design. Il s'agit maintenant de débusquer ce qui se cache derrière un tel lieu commun, pour mieux caractériser et neutraliser ce qui s'annonce comme une véritable trahison de la graphique opérée par ce projet de design.

\section{Design-moi une donnée...(data-design)}

Juxtaposer design et data pour composer ce récent datadesign n'est pas une simple substitution de la traditionnelle visualisation par une équivalence jugée plus contemporaine. Nous la tenons pour une commutation effective dans le fait que ce changement d'expression opère également un profond changement de contenu (énoncé) et de point de vue (énonciation). Ce design invite, selon Beyaert-Geslin (2012:3-4), à un double dépassement du rapport qui lie forme et fonction. Le premier déplace la question de la fonction de la visualisation vers les fonctions et valeurs, installant d'emblée un espace de la stratégie dans lequel cohabitent plusieurs objectifs et systèmes de valeurs potentiellement concurrents. L'horizon des références devient alors instable et sujet à négociations, contrastant avec la référence toute puissante d'une fonction 
unique, fixée de manière apriorique. Le deuxième déplacement concerne le rapport entre l'immatérialité d'un projet, pensé comme préfiguration, et la matérialité des objets qu'il est censé transformer. Pour dépasser cette vision unilatérale de la réalisation, Beyaert-Geslin (2012 : 3-4) propose de passer du surplombant surdesign ou métadesign à un redesign qui s'appuierait sur les propriétés de l'existant, prenant en charge la fabrique de la vie, l'améliorant précautionneusement. La diversification des fonctions de la visualisation des données transparaît dans les définitions les plus communes du design. Le dictionnaire en ligne Larousse définit par exemple ce qui est design comme ce qui est doté d'un " modernisme fonctionnel sur le plan esthétique " 5 . Autrement dit, nous identifions bien comment le design associe à la valeur informationnelle initiale, un réseau de valeurs esthétiques et rhétoriques (le modernisme). La préfiguration textuelle de la graphique laisse donc place à une anticipation plus floue : celle d'un montage stratégique stabilisé que nous pourrions dénommer signe réseau 6 .

\section{Le design esthétique}

Par le titre de son ouvrage Information is beautiful, McCandless (2012) affirme que la visualisation d'information manifeste une certaine beauté. Ce facteur esthétique se limite le plus souvent à une séduction (attractiveness) de la solution choisie, ce que dénoncent Vande Moere et Purchase : "L'attrait fait référence à ce que nous avons appelé jusque-là «esthétique» : le charme ou la beauté d'une solution donnée $»^{7}$. Au contraire, celles qu'ils nomment les esthétiques informationnelles ${ }^{8}$, ne se limitent plus à des effets émotionnels, mais se présentent comme des facteurs de réussite du montage visuel. Elles influencent positivement les performances et la satisfaction des usagers (Norman, 2002), améliorent la mémorisation et la justesse d'interprétation (Baterman et al., 2010). Cependant, d'un point de vue méthodologique, nous buttons contre un jugement esthétique qui ne peut être objectivement mené qu'à l'aune des effets sur la performance interprétative et l'attractivité. Mesurer la qualité esthétique n'est pas un acte trivial, selon Vande Moere et Purchase (2011), car il n'y a pas de principes esthétiques communs quantifiables et étalonnables, ce qui expliquerait pourquoi le facteur esthétique apparaît largement sous-représenté dans les recherches académiques. Toutefois, nous pouvons faire un pas de plus en liant une réflexion de Vande Moere et Purchase à l'approche du design de Beyaert-Geslin. Ce lien, nous le trouvons

5 Dictionnaire Français en ligne - Larousse, http://www.larousse.fr/dictionnaires/francais-monolingue, consulté le 25 avril 2014.

6 L'expression est empruntée à Stockinger. Voir Peter Stockinger, Traitement et contrôle de l'information. Procédures sémiotiques et textuelles, Paris, Hermes Science Publications, 2001.

7 Notre traduction de : «Attractiveness refers to what we have hitherto called 'aesthetics': the appeal or beauty of a given solution ", cf. Andrew Vande Moere et Helen Purchase, "On the role of design in information visualization ", Information Visualization, vol. 10, n 4, octobre 2011, p. 356-371.

8 Notre traduction d'information aesthetics. 
dans l'ouverture proposée par les deux premiers chercheurs : "L'esthétique ne se limite pas à la forme visuelle, mais inclut également des aspects flous tels que l'originalité, l'innovation et la nouveauté ainsi que d'autres facteurs plus subjectifs prenant en compte l'expérience de l'utilisateur »". L'originalité, l'innovation et la nouveauté constituent alors, à nos yeux, la dimension rhétorique du design d'information.

\section{Le design rhétorique}

Selon Quintilien, rhéteur du $1^{\text {er }}$ siècle après J.-C., la figure de rhétorique est « un changement raisonné du sens ou du langage par rapport à la manière ordinaire et simple de s'exprimer » (Quintilien, Cousin et Jal, 1978 : 11-13). Dans la perspective plus récente du Groupe $\mu$ (1992 : 256), la rhétorique se laisse définir de manière restreinte comme un écart (allotopie) vis-à-vis des règles ou conventions d'un discours (isotopie), donnant lieu, du point de vue de l'interprétation, à la mise en relation d'un degré perçu avec un degré conçu. Un tel fonctionnement amène alors le récepteur " 1) à considérer l'énoncé comme non acceptable (non grammatical), et 2) à y projeter un tel degré conçu » (Groupe $\mu 1992$ : 256). Saemmer (2011 : 23) a notamment reconnu cette conception de l'écart rhétorique dans l'abus chez Paul Valery, le viol pour Jean Cohen, le scandale de Roland Barthes, l'anomalie de Tzvetan Todorov ou encore la déviation chez Léo Sptizer et la subversion de Nicolas Beauzée.

Dans notre cas précis, les chercheurs mettent l'accent sur une dimension rhétorique du design conçue comme une nouveauté en rupture avec les normes ou conventions de l'ancien. Comme le dit Beyaert-Geslin (2012:16), les objets de design «ne sont censés être ni plus beaux ni plus fonctionnels mais incarnent seulement cette nouveauté se suffisant à elle-même ». La chercheuse joue alors de l'article défini ou indéfini pour illustrer l'opération rhétorique. Dans notre cas, la visualisation comme occurrence manifestée désavoue en certains points une visualisation conçue comme un prototype. Ce désaveu partiel du prototype par l'occurrence révèle les contraintes implicites du genre et les questionnent. Du côté de l'observateur, cette impertinence devient rhétorique à partir du moment où elle reste acceptable selon les standards culturels. En effet, l'écart rhétorique amuse s'il reste un jeu de langage innovant, bousculant les codes de manière à marginaliser la production.

\section{Design-moi les données sur les moutons...}

Revenant à Paukner, nous pouvons identifier comment il re-design la chronique pour y valoriser une signification esthétique et rhétorique. Du point de vue de la beauté, la diminution des repères en abscisse et en ordonnée procure un allégement de la représentation. Les formes obéissent à un certain

9 Notre traduction de : « aesthetics does not limit itself to the visual form, but also includes fuzzy aspects such as originality, innovation and novelty, and other subjective factors comprising the user experience " (Van de Moore et Purchase, 2011). 
minimalisme, elles sont plus pures, plus dégagées. Le fond blanc original laisse place à un bleu nuit qui souligne, par contraste, les courbes supportant l'information principale. Le ton parme du dispositif de la chronique manifeste un certain effet de fondu, une adhésion au fond qui révèle la mise à l'arrièreplan du standard graphique pour poser les courbes au-devant de la scène. Ces dernières présentent pour cela des teintes lumineuses et saturées tranchant clairement avec le fond. Reprenant une expression de Jeanneret (2001), nous pourrions dire que ce dispositif complexe chercherait à faire passer pour une évidence informationnelle un discours persuasif et manipulatoire. En effet, les courbes manifestent un mensonge dès que leur forme n'est plus déterminée par les seules données (data-driven). Les séries de lignes horizontales, habituellement parallèles, s'écartent avec le déplacement de gauche à droite au seul bon-vouloir du designer (design-driven). Un œil avisé, doté de compétences interprétatives repère rapidement la transgression du genre. Par une alphabétisation informationnelle (une littératie?), l'observateur devient capable de faire la part entre les déformations liées aux données et celles imputables au style du designer. Ainsi révélé, le standard aurait souhaité la conservation du parallélisme et l'écart visuel devient donc écart rhétorique. Il dynamise le contenu atone du parallélisme, il le remet en question pour le resémantiser. Nous comprenons alors que ce relâchement des lignes accompagne également celui du stéréotype, acte rhétorique inaugural pour dire autre chose. À un premier niveau d'interprétation, cet écartement ne dénote rien d'autre qu'un effet de zoom progressif puisque la transformation géométrique est homothétique (agrandissement proportionnel). Cependant il engendre une première dissonance cognitive induisant en erreur l'observateur lorsque la courbe, ainsi agrandie, s'aplatit pour donner l'impression d'une stagnation des effectifs : les légendes accompagnant le pic de 1982 et la valeur finale de 2011 contredisent l'évidence visuelle. Cependant, cette exception dans la lecture d'ensemble, que constituent les ronds du pic et de la dernière valeur (lecture élémentaire), trahirait une primauté du souci de vérité de l'information sur les effets persuasifs des choix esthétiques du design. Seulement, si l'évocation des remords du designer prête à sourire, c'est que son design n'est pas destiné à servir le mensonge, mais bien à tout autre chose : par cette déformation, il cherche à nous raconter une histoire.

\section{Raconte-moi une histoire sur les données...(data-storytelling)}

S'amorce alors une spécialisation du discours visuel, intégrant au projet une dimension narrative. Cependant, nous n'avons pas ici les moyens de développer une définition consistante de la notion, étant donné les nombreuses théories auxquelles elle donna lieu (citons par exemple Barthes, Greimas, Bremond, Eco, Genette, Metz, Todorov). Aussi, nous restreindrons drastiquement la narrativité à l'expression d'une transformation telle que décrite dans le programme narratif de Greimas (1969). Dans nos représentations visuelles, elle 
se manifestera par la mise en scène d'une action reliant une situation initiale à une situation finale, dont les contenus respectifs seront inversés (Fontanille, 1999 : 198-199). Segel et Heer proposent une définition du narratif qui rejoint celle de la transformation, insistant sur la notion de séquence - pour nous les états initial et final consécutifs - et de transition - pour nous l'action : "Ils ont développé des techniques subtiles pour diriger séquentiellement l'attention du spectateur et la maintenir orientée au-delà des transitions $»^{10}$. Cette transformation, quoique sommaire, paraît suffire à rendre compte du narratif dans les projets de data-storytelling qui distinguent clairement design et récit comme l'expriment Spaulding et Faste : « Nous croyons dans le fait qu'un prototypage efficace des éléments diégétiques nécessite une attention également répartie entre le design et la trame narrative $»^{11}$. Le problème pour le designer se pose alors en termes de conversion de l'histoire à raconter en un design spatial ${ }^{12}$. À un niveau superficiel, nous ne pouvons manquer l'exposition d'un élément figuratif, celui du mouton blanc situé en haut à droite du graphique. D'un point de vue plastique, un lien chromatique - le blanc - a été mobilisé, rapprochant sémantiquement le mouton figuré de la courbe de leur population. Deuxième élément figuratif, la présence typique d'une barrière constituée de trois poteaux et d'un cordage. Enfin, ces éléments composent une scénette en cours, celle d'un mouton sautant la barrière. Seulement, de cette description rapide, nous pouvons tirer deux histoires racontées : celle des moutons et celle du design.

\section{Raconte-moi une histoire sur le mouton de Nouvelle-Zélande...(le discours narratif)}

La première histoire rend compte de la supériorité du nombre de moutons sur la population humaine. La dimension narrative se présente donc comme une redondance figurative d'un thème déjà évoqué par la chronique dont il est le déploiement figuratif. En revanche, si la barrière demeure absente de la chronique, celle-ci permet de rejouer une action plus générale : le dépassement. Cette petite scénette engendre l'analogie, puisque la barrière et la population humaine sont interchangeables, et manifeste ainsi une redondance diagrammatique. Par ailleurs, le très sérieux site DataMarket, propose dans son titre une bien curieuse question pour qui veut se conformer aux exigences de la graphique : "Man vs sheep: a New-Zealand myth? ». Ce

\footnotetext{
10 Notre traduction de : « they have developed nuances techniques for sequentially directing a viewer's attention and keeping viewers oriented across transitions ", cf. Edward Segel et Jeffrey Heer, « Narrative visualization: telling stories with data ", IEEE transactions on visualization and computer graphics, 2010.

11 Notre traduction de : "we believe that effectively prototyping diegetic design concepts requires equal attention on both the design and the story », cf. Eric Spaulding et Haakon Faste, « Design-driven Narrative: Using Stories to Prototype and Build Immersive Design Worlds ». In Proceedings of the SIGCHI Conference on Human Factors in Computing Systems, 2013, p. 2843-2852. ("CHI'13 »)

12 Notre traduction de spatial design dans Edward Segel et Jeffrey Heer, « Narrative visualization: telling stories with data ", op. cit.
} 
qui confirme, même dans ce site, la force d'un mythe déjà-là à propos du pays que les données statistiques rendent véridique et font accéder au rang de fait scientifiquement attesté. Quant à Paukner, il intitule sa visualisation «Dominated by sheep» en ne manquant pas d'ajouter une petite couronne sur le $\mathrm{O}$, insistant sur le comique de la supériorité du mouton qui règne sur le pays.

\section{Raconte-moi une histoire sur le design d'information...(le métadiscours narratif)}

La deuxième transformation racontée est celle d'un standard graphique - la chronique - en un standard figuratif - la barrière. Lorsque Paukner convertit sous nos yeux un objet graphique en une sémiotique du monde naturelle, l'histoire se concentre sur la manière de dire les choses (l'énonciation). Cette narration met donc en scène une transformation concernant le discours luimême et pour cette raison nous la considérons comme métadiscursive. Paukner montre comment il se libère du standard de la graphique pour le remanier, le resémantiser en exposant du même coup son savoir et son savoir-faire créatif, mêlant nouveauté et prudence. Pour cela, il use du sens conventionnel de la lecture occidentale pour opposer une situation initiale - à gauche - d'une chronique conforme à la graphique et une situation finale - à droite - par la présence d'une sémiotique figurative, transformant les lignes pointillées en des cordages (utilisation de serpentins réguliers) et l'axe des ordonnées (inutilement répété selon la graphique) en d'ingénieuses représentations des poteaux.

Cette transformation présente, comme toute histoire racontée, une certaine orientation discursive. Il ne s'agit pas d'une barrière devenant chronique mais bien l'inverse. Or, cette orientation est en réalité le résultat d'une décision de la part du designer car d'autres, comme Peter Ørntoft, exemplifient la démarche inverse. Dans ses graphiques, intitulés «Refugees and immigrants " (Klanten et al., 2011), nous voyons comment dans différentes photographies, une kippa, un hijab et un chapelet catholique deviennent chacun un diagramme circulaire. Ici, c'est la sémiotique du monde naturel qui exprime alors sa diagrammaticité, au service d'un discours sur des données statistiques.

\section{Conclusion}

Cette étude nous a permis de dégager des dimensions constituantes d'un genre récent, celui du design et du récit de données (data-storytelling). Ces dimensions permettent de reconstituer un fond de culture préfigurative qui fonctionne comme un système de régulation des activités par un groupe d'acteurs (Stockinger, 2001). Cependant, nous pourrions aller plus loin en distinguant dans ces activités deux grandes tendances dans la conception d'une préfiguration : en référence à Boutinet (2012), nous différencions une conception existentielle et une conception rationalisante. 
La première fixe des normes informationnelles, esthétiques, rhétoriques et narratives sous la forme d'un vouloir-formel précis qui rencontre, pour seule inconnue, le mode opératoire. Autrement dit, le designer obéit à une fin qui justifie les moyens. La seconde privilégie le respect d'un mode opératoire conçu comme une "dimension générale, de nature normative et sociale, transmise par la tradition et l'apprentissage » (Fontanille, 2008 : 233). Cette déontologie oblige donc le designer à un fais ce que tu dois, advienne que pourra. Aussi, nous avons remarqué comment le projet concret naviguait entre ces deux extrêmes théoriques, entre le purpose - le projet-dessein et le project - le projet-programme, de manière à engendrer une anticipation intermédiaire, plus floue et partiellement déterminée (Boutinet, 2012).

Quand Paukner vise un objectif rhétorique de renouvellement du langage visuel, nous remarquons comment il manifeste une certaine prudence avec les standards dès lors qu'il veille à maintenir une certaine vérité de l'information. Il présente une certaine éthique d'ordre déontologique, vis-à-vis de la graphique, et plus implicitement une hiérarchisation des dimensions. Autrement dit, par ce positionnement éthique perceptible dans la représentation visuelle, le designer se fie à une autre référence : lui-même. Il s'auto-convoque pour dresser une déontologie individuelle - une caractérologie (Fontanille, 2008 : 234) permettant de lier ensemble des déontologies potentiellement concurrentes.

Avant de clore le propos, nous pourrions bien sûr évoquer une hypothèse concernant l'orchestration discursive (Stockinger, 2001). En effet, notre visualisation doit respecter certaines contraintes taxonomiques, car la visualisation doit à la fois être reconnue comme chronique, comme design et comme récit. Pour cela elle doit s'accommoder des différentes normes méréologiques (la manière de construire des totalités) qui organisent une scène-d'un-mouton-sautant-la-barrière (thématique), une scène-devisualisation-d'information et une scène narrative. La médiation entre toutes ces scènes semble se situer dans les traits figuratifs communs. En effet, si la scène du mouton s'intègre parfaitement dans la chronique, c'est parce que cette dernière contenait en elle des caractéristiques formelles compatibles, un noyau narratif et figuratif que le designer a su exploiter et déployer. Ainsi, le designer mobilise le caractère polysémique des formes pour créer du lien entre les contraintes et faire émerger un signe stable : un signe réseau (Stockinger, 2001).

\section{BIBLIOGRAPHIE}

BATEMAN Scott, MANDRYK Regan L., GUTWIN Carl, GENEST Aaron, MCDINE David et BROOKS Christopher (2010), «Useful junk?: the effects of visual embellishment on comprehension and memorability of charts ", Human Factors in Computing Systems (CHI'10), p. 2573-2582. 
BERTIN Jacques (1981), «Théorie matricielle de la graphique », Communication et langages, vol. $48, \mathrm{n}^{\circ} 1$, p. 62-74.

BERTIN Jacques (2013), Sémiologie graphique: les diagrammes, les réseaux, les cartes, Paris, Éd. de l'EHESS.

BEYAERT-GESLIN Anne (2012), Sémiotique du design, Paris, PUF.

BOUTINET Jean-Pierre (2012), Antbropologie du projet, Paris, PUF.

FONTANILLE Jacques (1999), Sémiotique du discours, Limoges, Presses Universitaires de Limoges (« Nouveaux Actes Sémiotiques »).

FONTANILLE Jacques (2008), Pratiques sémiotiques, Paris, PUF (« Formes sémiotiques ").

FONTANILLE Jacques (2011), Corps et sens, Paris, PUF (« Formes sémiotiques »).

FONTANILLE Jacques et DONDERO Maria Giulia (2012), « Problèmes narratifs et solutions iconiques : le statut sémiotique des courbes mathématiques ». In Des images à problèmes - Le sens du visuel à l'épreuve de l'image scientifique, Limoges, Presses Universitaires de Limoges, p. 207-238.

FRIENDLY Michael et DENIS Daniel J. (2014), Milestones in the history of thematic cartography, statistical graphics, and data visualization, http://www.datavis.ca/milestones/, consulté le 11 avril 2014.

GREIMAS Algirdas Julien (1969), « Éléments d'une grammaire narrative ", L'Homme, vol. 9, n 3, p. 71-92.

GREIMAS Algirdas Julien et COURTÉS Joseph (1993), Sémiotique : Dictionnaire raisonné de la théorie du langage, Paris, Hachette Éducation.

GROUPE $\mu$, EDELINE Francis, KLINKENBERG Jean-Marie et MINGUET Philippe (1992), Traité du signe visuel: Pour une rhétorique de l’image, Paris, Seuil («La couleur des idées ").

HJELMSLEV Louis (1971), Essais linguistiques, Paris, Minuit (« Arguments »).

JEANNERET Yves (2001), «Les politiques de l'invisible : Du mythe de l'intégration à la fabrique de l'évidence », Document numérique, vol. 5, n 1, p. 155-180.

KLANTEN Robert, EHMANN Sven et SCHULZE Floyd (2011), Visual Storytelling, Berlin, Gestalten.

MCCANDLESS David (2012), Information is Beautiful, New Edition, New-York, Collins.

NORMAN Don (2002), «Emotion \& Design: Attractive Things Work Better », Interactions, Vol. 9, $\mathrm{n}^{\circ}$ 4, juillet, p. 36-42.

PAUKNER Michael (2013), «Des ovins et des hommes », Courrier International, mai, p. 39.

PAUKNER Michael (2014), Dominated by sheep, http://www.substudio.com/ dominated-by-sheep, consulté le 25 avril 2014.

QUINTILIEN, Cousin Jérôme et JAL Paul(1978), De l'institution oratoire, tome 5 : Livres VIII-IX, Paris, Les Belles Lettres.

RASTIER François (2001), Arts et sciences du texte, Paris, PUF.

SAEMMER Alexandra (2011), «De la confirmation à la subversion : Les figures d'animation face aux conventions du discours numérique », Protée, vol. 39, n 1, p. 23-36. 
SEGEL Edward et HEER Jeffrey (2010), « Narrative visualization: telling stories with data ", IEEE transactions on visualization and computer graphics, p. 1139-1148.

SPAULDING Eric et FASTE Haakon (2013), « Design-driven Narrative: Using Stories to Prototype and Build Immersive Design Worlds ». In Proceedings of the SIGCHI Conference on Human Factors in Computing Systems, p. 2843-2852. ("CHI '13 »)

STOCKINGER Peter (2001), Traitement et contrôle de l'information. Procédures sémiotiques et textuelles, Paris, Hermes Science Publications.

VANDE MOERE Andrew et PURCHASE Helen (2011), «On the role of design in information visualization ", Information Visualization, Vol. 10, n 4, october, p. 356-371.

Résumé : Adoptant une entrée stratégique, le projet devient une préfiguration associant plusieurs standards potentiellement concurrents. Un genre comme le data-storytelling permet d'observer cette négociation opérée entre plusieurs dimensions visuelles. Dans une perspective génétique, nous étudierons comment une data-visualisation, conforme à la graphique de Bertin, peut laisser place à un data-design intégrant la beauté (esthétique) et la nouveauté (rhétorique), puis à un data-storytelling mobilisant une transformation narrative. L'article examinera un cas représentatif, celui d'une œuvre de Michael Paukner intitulée Dominated by sheep évoquant les effectifs ovins en Nouvelle-Zélande et faisant écho à une mythique prise de pouvoir du mouton néo-zélandais.

Mots-clés : projet, visualisation d'information, design d'information, récit de données.

\begin{abstract}
Considering a strategic point of view, the project/purpose becomes a prefiguration associating potentially rivals points of view. Visual data-storytelling allows us to observe this negotiation operated between four dimensions. In a genetic perspective, we shall study how datavisualization, in compliance with Bertin's graphics, can give way to data-design, integrating the beauty (esthetics) and the novelty (rhetoric), then to one data-storytelling, mobilizing narrative processing. The article will examine a representative case, that of Michael Paukner's work intitled "Dominated by sheep". It refers to the ovine population in New Zealand and recounts the mythical superiority of sheep in that country.
\end{abstract}

Keywords: project, information visualization, information design, data-storytelling. 
